OPEN ACCESS

Edited by:

Serk in Park

Korea University, South Korea

Reviewed by:

Yeliz Guven,

Istanbul University, Turkey

Jan Kühnisch,

LMU Munich University

Hospital, Germany

${ }^{*}$ Correspondence:

Niki M. Moutsopoulos

nmoutsop@mail.nih.gov

Specialty section: This article was submitted to

Pediatric Dentistry,

a section of the journal

Frontiers in Dental Medicine

Received: 22 February 2021

Accepted: 13 April 2021

Published: 12 May 2021

Citation:

Brenchley L, Ferré EMN, Schmitt MM,

Gardner PJ, Lionakis MS and

Moutsopoulos NM (2021) Case

Report: Dental Findings Can Aid in

Early Diagnosis of APECED

Syndrome.

Front. Dent. Med. 2:670624.

doi: 10.3389/fdmed.2021.670624

\section{Case Report: Dental Findings Can Aid in Early Diagnosis of APECED Syndrome}

\author{
Laurie Brenchley ${ }^{1}$, Elise M. N. Ferré ${ }^{2}$, Monica M. Schmitt ${ }^{2}$, Pamela J. Gardner ${ }^{3}$, \\ Michail S. Lionakis ${ }^{2}$ and Niki M. Moutsopoulos ${ }^{1 *}$
}

${ }^{1}$ Oral Immunity and Inflammation Section, National Institute of Dental and Craniofacial Research, National Institutes of Health, Bethesda, MD, United States, ${ }^{2}$ Fungal Pathogenesis Section, Laboratory of Clinical Immunology and Microbiology, National Institute of Allergy and Infectious Diseases, National Institutes of Health, Bethesda, MD, United States, ${ }^{3}$ Dental Consult

Services, National Institute of Allergy and Infectious Diseases, National Institutes of Health, Bethesda, MD, United States

Autoimmune polyendocrinopathy-candidiasis-ectodermal dystrophy (APECED), also known as autoimmune polyglandular syndrome type 1 (APS-1), is a rare genetic disorder caused most often by biallelic mutations in the AIRE gene. Classic clinical findings of the disease are chronic mucocutaneous candidiasis and autoimmunity that primarily targets endocrine tissues, such as hypoparathyroidism and adrenal insufficiency. Recently, however, it has been appreciated that enamel hypoplasia, together with intestinal malabsorption and a characteristic APECED rash, is a prominent early disease manifestation of APECED which can aid in the diagnosis of disease before other potentially life-threatening disease manifestations occur. To demonstrate this point, we present data from a cohort of APECED patients, 70\% of who present with enamel dysplasia at an early age. Importantly, early life presentation with enamel dysplasia was predictive of likelihood for subsequent APECED diagnosis. Furthermore, we present a case of a patient with APECED and severe enamel defects and discuss the utility of medical-dental professional co-operation in the diagnosis and management of this complex disorder.

\section{Keywords: APECED, APS-1, AIRE, primary immune deficiency, chronic candidiasis, enamel hypoplasia}

\section{INTRODUCTION}

A growing body of evidence has suggested an association between oral manifestations and systemic disease $(1,2)$. Indeed, immunocompromised patients will present with various oral infection susceptibility. Often patients with numerous systemic, cutaneous and gastrointestinal inflammatory symptoms will also have clinical oral manifestations. It is therefore imperative that medical and dental professionals strengthen a collaborative understanding of common risk factors and biological findings related to oral and systemic disease. This article features clinical and diagnostic criteria and oral manifestations of Autoimmune polyendocrinopathy-candidiasis-ectodermal dystrophy (APECED), also known as Autoimmune polyglandular syndrome type 1 (APS-1) to highlight the importance of a multidisciplinary approach in providing early diagnosis and comprehensive care to patients. 


\section{OVERVIEW OF APECED}

APECED is an inherited monogenic disorder caused most often by biallelic mutations in AIRE and results in the development of multi-organ autoimmunity, chronic mucocutaneous candidiasis (CMC), and ectodermal dystrophy. AIRE is a transcription regulator highly expressed in thymic medullary epithelial cells (mTECs) where it orchestrates negative selection of auto-reactive T cells (3). These cells escape into the periphery in the AIREdeficient patient where they are both necessary and sufficient for development of autoimmunity (4-6).

APECED affects $\sim 1: 100,000-1: 200,000$ people internationally with higher prevalence in Iranian Jews, Sardinians, Finns and Slovenians $(\sim 1: 9,000-1: 25,000)$ and lower prevalence in populations of Norwegian $(1: 80,000)$, Irish $(1: 130,000)$, French $(1: 500,000)$ and Japanese descent $(1: 10,000,000)$. In the U.S., APECED is estimated to occur in 1:100,000-1:300,000 persons (7-9).

More than 25 disease manifestations (shown in Table 1) have been reported through the course of disease $(5,10)$ amongst which CMC, hypoparathyroidism, and adrenocortical failure (Addison's disease), are three of the most highly prevalent. Together they form the "classic triad" and development of any two (a "diagnostic dyad") of these is sufficient for establishing a clinical diagnosis of $\operatorname{APECED}(6,11)$. Although this classic triad is characteristic for APECED, it often occurs late in the course of the disease. Through the comprehensive evaluation of our APECED cohort at the National Institutes of Health

TABLE 1 | Clinical manifestations that affect patients with APECED.

\section{Manifestations of APECED}

- Chronic mucocutaneous candidiasis (CMC)

- Enamel hypoplasia

- Sjogren's-like syndrome

- Aphthous ulcers

- Urticarial eruption

- Alopecia

- Vitiligo

- Nail dystrophy

- Retinitis

- Keratoconjunctivitis

- Hypoparathyroidism

- Adrenal insufficiency (Addison's disease)

- Growth hormone deficiency

- Autoimmune gastritis

- Gastric carcinoma

- Asplenia

- Intestinal dysfunction

- Tubulointerstitial nephritis

- Autoimmune hepatitis

- Autoimmune pneumonitis

- Pernicious anemia

- Iron deficiency

- Type 1 diabetes

- Pure red cell aplasia

- Hypogonadism

- Asplenia

- Hypothyroidism
(NIH) Clinical Center, we found $80 \%$ of patients developed an average of 3 non-classic triad manifestations before reaching a diagnostic dyad (10). Amongst these, enamel hypoplasia, intestinal malabsorption, and a characteristic APECED rash were the most prevalent early clinical manifestations. In fact, their inclusion in expanded diagnostic criteria (classic triad + adjunct triad) (Figure 1) would have reduced the time to diagnosis by half (Figure 2) (10), which has potential major implications in earlier recognition of the disease and could help improve patient outcomes.

These findings call for a multidisciplinary approach to patient evaluation with participation of multiple specialties of medicine and dentistry. Due to advances in sequencing technology, gene mutations are more frequently identified and support the theory that an inheritable factor exists in this disorder. However, identification through genetic testing is not always definitive or clinically available. There is a wide range and variability of disease manifestations and phenotypic expression of APECED (Table 1). Clinicians including endocrinologists, allergists, pediatricians, gastroenterologists, hematologists, ophthalmologists, pulmonologists, dermatologists, dentists and dental hygienists, can all recognize early-onset symptoms and disease manifestations and begin a differential diagnosis that may lead to early interventions helpful in establishing an APECED diagnosis and preventing life-threatening complications.

\section{ENAMEL DEFECTS CAN BE AN EARLY SIGN OF APECED}

Enamel defects may be a phenotype of hereditary conditions that involve only the enamel matrix or may be a component of a hereditary medical syndrome. Enamel is formed by ameloblasts during tooth development (12). This protective outermost layer of the tooth structure helps protect the inner dentinal and pulpal tissues from masticatory forces and continual bacterial and other pathogenic insults. Ameloblast disruptions during the development of the enamel matrix can result in hypoplastic and hypo-mineralized enamel (12). Hereditary syndromes such as Treacher Collins, which presents with craniofacial abnormalities of the zygomatic complex, jaws and palate $(13,14)$; Heimler syndrome, characterized by sensorineural hearing loss and nail abnormalities $(15,16)$; and Usher syndrome, which features sensorineural hearing loss and retinitis pigmentosa (17) have all reported enamel hypoplasia in clinical case reports.

Consistent with a previous report, but in an expanded cohort, we found enamel hypoplasia in $70 \%$ of 104 patients with APECED in our cohort. Moreover, with expansion of the diagnostic criteria for APECED (with use of the aforementioned adjunct triad), we find that CMC, APECED rash and hypoparathyroidism appear most frequently in combination with enamel hypoplasia early in life (Table 2). These findings may be an early predictor of those who are more likely to develop additional life-threatening manifestations of disease later in life (Figure 2). 


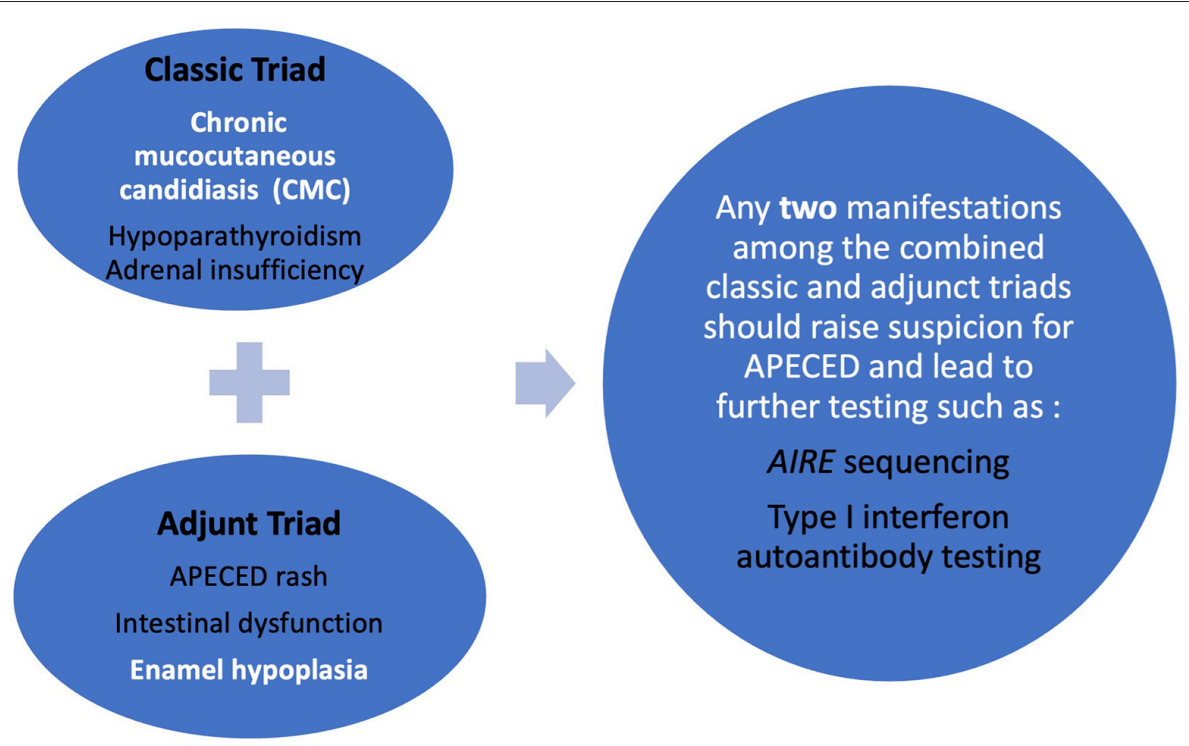

FIGURE 1 | Refined diagnostic criteria (combined classic and adjunct triad) designed to enhance earlier diagnosis of APECED.
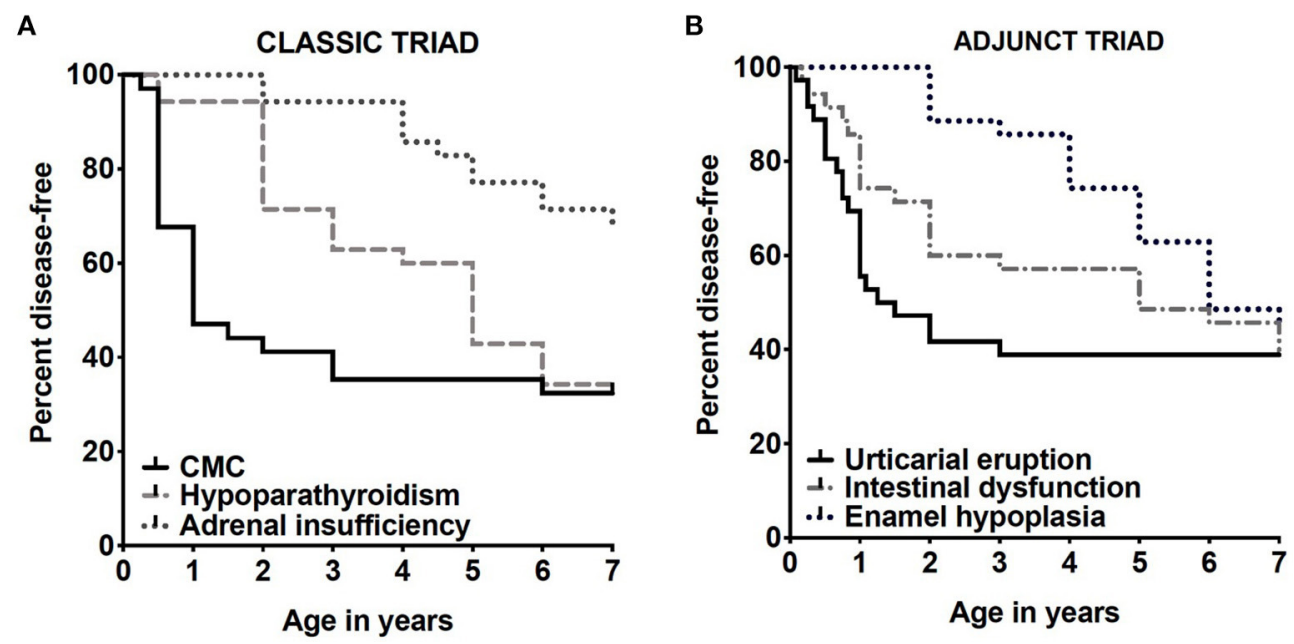

FIGURE 2 | Shown are Kaplan-Meier curves demonstrating prevalence by age of classic triad manifestations (A) and adjunct triad (B) appearing by age 7 years, the average age of diagnosis by classic diagnostic criteria among the first 35 patients with APECED. These images are derived from Ferre et al. (10).

\section{CASE DESCRIPTION}

Here we present a 15-year-old Caucasian male of European descent with confirmed biallelic AIRE gene mutations enrolled in protocol NCT01386437 who presented to the NIH dental clinic with concerns regarding the esthetics of his smile. His medical history included the appearance of an intermittent rash during his first year of life that resembled flat red macules that could not be explained by the pediatrician at that time; reflecting back to the presentation, this rash was one of the early primary clinical indicators of APECED. During his third year of life, he presented to his physician with the development of alopecia and finger/toenail dystrophy. In these very early years, the patient was otherwise clinically well. However, by his sixth year he began suffering from abdominal pain which led to an emergent visit to the emergency room. During this visit the patient presented with significant abdominal discomfort, fatigue, increased thirst and salt cravings and was found to have severely low sodium levels at $115 \mathrm{mmol} / \mathrm{L}$ leading to diagnosis of adrenal insufficiency.

In addition, his dental history and exam revealed that he had several tooth extractions due to breakdown of the clinical crown; a note of enamel hypoplasia was made. Over the next 3 years other clinical complications included gallstones, vitamin $\mathrm{D}$ deficiency and iron deficiency. He underwent a liver biopsy at age 10 for persistently elevated transaminases and was diagnosed with autoimmune hepatitis, a condition 
TABLE 2 | Frequency of diseases appearing in combination with enamel hypoplasia as the first two consecutive clinical manifestations in 35 patients with APECED.

\begin{tabular}{lc}
\hline Clinical manifestation & Number (\%) of patients \\
\hline CMC & $1(3)$ \\
APECED rash & $1(3)$ \\
Hypoparathyroidism & $1(3)$ \\
\hline
\end{tabular}

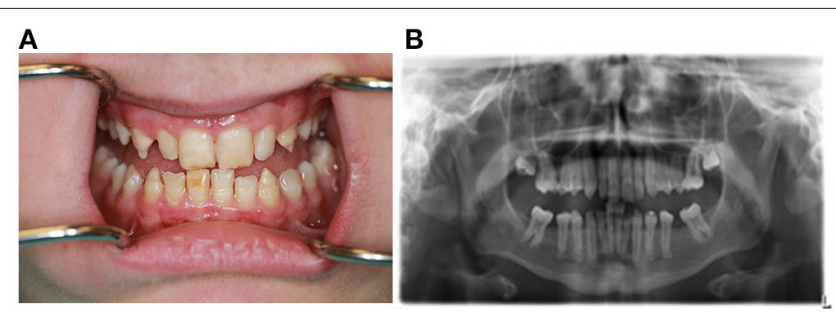

FIGURE 3 | Clinical intraoral photograph and panoramic radiograph of 15-year-old Caucasian male APECED patient demonstrating abnormal dental development with enamel hypoplasia (photo, A), taurodontism (radiograph, B).

affecting $\sim 40 \%$ of APECED patients (18). At this point, APECED was suspected prompting AIRE sequencing, which identified homozygous c.967_979del13 deletions. Importantly, he did not have any history of CMC or hypoparathyroidism at that time, which reiterates the heterogeneity of clinical manifestation development and disease progression of APECED.

The dental findings in this case depict developmental anomalies associated with APECED that can be detected with routine dental examinations in early age (Figure 3). Upon examination extra-orally, there was no palpable lymphadenopathy. Intraorally, the patient presented with adult dentition that exhibit dental manifestations of APECED including irregular clinical crown size with pointy cusp tips and narrow shape. The buccal tooth enamel was chalky white and yellow with horizontal grooves and pitting of the enamel surfaces. The cusp tips were white, and the remaining enamel was thin and opalescent. There was a deep bite with an overbite of $100 \%$. There was spacing between the teeth which may have been because of the narrower teeth, but also the patient was missing all maxillary and mandibular first permanent molars. In addition, radiographic findings reveal taurodontism of all maxillary and mandibular second molars. There was no clinical or radiographical evidence of caries or oral candidiasis.

\section{DISCUSSION}

When treating patients with APECED, co-operation of a multidisciplinary team is imperative. While APECED is commonly diagnosed by gene sequencing, not all patients with clinical APECED have biallelic AIRE mutations $(5,7,10)$. Some patients may have large AIRE deletions, or non-coding gene variants, or non-AIRE gene variations $(5,10)$. Importantly, genetic confirmation will typically follow the disclosure of clinical findings which will suggest the diagnosis of APECED. Therefore, early detection of oral manifestations can be fundamental for a targeted differential diagnosis.

Enamel hypoplasia is an important early clinical sign that can aid in diagnosis. It is a common finding in the permanent dentition among those that have been diagnosed with APECED, however, it was a less reported observation in deciduous teeth until the recent reporting of enamel hypoplasia, occurring in $17.3 \%$ of the cohort of patients at NIH. In fact, based on this recent study it is the first or second clinically reportable manifestation and overall 5th most common occurring manifestation of APECED (10). In addition, at least one scanning electron microscopy study evaluating the prismatic structure of enamel in patients with confirmed AIRE gene mutations has demonstrated compromised enamel matrix structures in deciduous and permanent teeth (19).

Children with APECED may present with patchy white-yellow discoloration on their teeth, or enamel hypoplasia which may cause the dentition to appear ridged and pitted or mis-shaped. Although APECED and other primary immunodeficiencies aforementioned are the focus of this paper, it does not go without mention that enamel hypoplasia has also been associated with prenatal abnormalities such as maternal vitamin D deficiency (20), low birth weight (21), or environmental factors such as trauma to the teeth during development (22) or systemic infection (23). Enamel hypoplasia in combination with another manifestation of the classic and adjunct triad should alert clinicians of a possible APECED diagnosis.

Early detection of oral developmental anomalies and caries is one of the primary reasons to have a child's first oral examination by 12 months of age. Defects of the enamel matrix predispose teeth to an increased risk of dental caries and erosion from acids in foods and beverages. Management of enamel hypoplasia in the primary dentition should focus on early diagnosis and preventive care. Routine topical fluoride application is one of the most effective anti-caries strategies as it helps reduce dentinal sensitivity, enamel remineralization, inhibits acid production and bacterial metabolism. In addition, treatment with other remineralizing agents such as casein phosphopeptide amorphous calcium phosphate (CPP-ACP) or silver diamine fluoride (SDF) can help re-mineralize the hypo-mineralized areas and early carious lesions on the tooth surface $(24,25)$. The patient we discuss in this case report did not present with active carious lesions at the time of their dental visit at our clinic. The patient was referred back to his primary care dentist for future treatment, with our suggestion to consider the mentioned anticaries strategies in the ongoing preventive care and treatment of this patient's dentition.

Patients with APECED will need preventive services with a 3-4 month interval so the oral status can be reviewed and evaluated. In cases of extensive enamel hypoplasia multidisciplinary team management involving general practitioners, specialist pediatric dentists and orthodontists may be necessary. A thorough dietary analysis in collaboration with the primary care physician and dietitian may assist with educating patients on how to improve their nutritional intake, 
especially of calcium and fluoride which are imperative to oral health.

Importantly, the diagnosis of enamel hypoplasia by oral health professionals may identify patients early in the course of the disease. In fact, two of the early clinical manifestations of APECED (enamel hypoplasia and oral candidiasis) can be identified by oral health professionals, suggesting a potential primary role for oral healthcare providers in disease diagnosis. CMC, commonly known as oral thrush, often occurs as an early primary symptom of APECED and can vary in appearance and severity. Thrush typically appears as a white coating on the tongue, oral mucosa, palate, gingiva, throat, or commissures of the mouth that can be wiped off with a gauze. Thrush can also present as redness and soreness that can prohibit eating spicy or acidic foods. Treatment for oral Candida infections includes topical antifungal agents such as miconazole, clotrimazole, amphotericin B or nystatin (5). APECED patients are also susceptible to Candida infections of the esophagus, vagina, and nails. Therefore, systemic treatments that may be considered also include fluconazole, voriconazole, posaconazole, the echninocandin antifungals or amphotericin B (5). Resistance can occur to oral antifungal agents which can make long-term management of CMC a challenge. Acute episodes should include a fungal swab culture with susceptibility testing to determine the pattern of in vitro susceptibility because different Candida strains can be differentially susceptible to antifungal therapeutics, and some strains may become resistant over time $(5,26)$. Treatment with antifungals lasting $4+$ weeks typically allows sufficient time of therapeutic intervention and reduces the potential for relapse. If left untreated Candida infections can lead to esophageal strictures or esophageal carcinomas $(26,27)$. However, additional novel treatments may be considered based on our recent findings in the pathogenesis of APECED-related mucosal candidiasis (28).

Beyond early diagnosis and management of early APECED signs, an oral healthcare provider may be also involved in the prevention and treatment of oral manifestations that may present throughout the disease process. The patient discussed in this case report did not present with any of the common oral symptoms at the time of exam. However, patients with APECED are at increased risk for xerostomia, and incidence of caries, aphthous ulcers, aphthous stomatitis, atrophic glossitis and importantly an increased risk of oral cancer due to chronic inflammation in association with CMC including oral and esophageal carcinomas (29). Sjogrens's-like syndrome may present early in life with a rise in incidence over time. Dry mouth symptoms can lead to discomfort and interfere with speech, eating and may contribute to burning mouth syndrome and increased caries risk.

Oral cancer screenings should be a routine part of every dental examination as alcohol intake and smoking are additional risk factors that may promote oral carcinogenesis (30). Awareness of patient social behaviors and risk as well as through documentation of oral candidiasis patterns and pseudomembranous lesions presenting with erosion, or ulceration noted during routine exams, can be helpful in the diagnosis of cancers at an early stage. Oral health professionals should instruct patients how to perform oral self-examinations and implement excellent oral hygiene, while stressing the importance of reporting any new or unusual oral symptoms and frequent dental follow up appointments. Oral candidiasis should be treated aggressively with antifungal therapies as mentioned earlier. While there are few guidelines for medications in managing oral ulceration, a primary objective is pain management to reduce and control symptoms, therefore adjunctive therapies include topical steroids and analgesics that can provide short-term pain relief to sooth aphthous ulcers (1). In cases where patients describe generalized oral sensitivity, use of an extra soft toothbrush and other recommendations such as elimination of spicy foods, carbonated beverages and toothpastes that contain sodium laurel sulfate may be suggested.

With increased screening, the recognition of APECED is growing especially in the US. Patients may seek dental care without knowing of their condition. Oral health professionals are well-positioned to note this disease's oral signs and symptoms. A thorough medical, family and dental history will aid in proper diagnosis and treatment. Oral health professionals who suspect APECED should speak with the patient's physician and or dietitian to determine an effective treatment strategy. This multidisciplinary approach will support the successful management of APECED as well as mitigate its effects on health.

\section{DATA AVAILABILITY STATEMENT}

The original contributions presented in the study are included in the article/supplementary material, further inquiries can be directed to the corresponding author.

\section{ETHICS STATEMENT}

The studies involving human participants were reviewed and approved by NIH IRB. Written informed consent to participate in this study was provided by the participants' legal guardian/next of kin.

\section{AUTHOR CONTRIBUTIONS}

LB and NM conceived and wrote the manuscript. LB and PG performed clinical work. LB, EF, MS, PG, ML, and NM were evolved in the study conception, data analysis, and interpretation. EF, MS, PG, ML, and NM critically edited the manuscript. All authors contributed to the article and approved the submitted version.

\section{FUNDING}

Funding for this study was provided by the Division of Intramural Research, National Institute of Dental and Craniofacial Research and the National Institute of Allergy and Infectious Diseases, NIH. The content of this publication does not necessarily reflect the views or policies of the US Department of Health and Human Services, nor does the mention of trade names, commercial products, or organizations imply endorsement by the US government. 


\section{REFERENCES}

1. Mays JW, Sarmadi M, Moutsopoulos NM. Oral manifestations of systemic autoimmune and inflammatory diseases: diagnosis and clinical management. J Evid Based Dent Pract. (2012) 12:265-82. doi: 10.1016/S1532-3382(12)70051-9

2. Moutsopoulos NM, Moutsopoulos HM. The oral mucosa: a barrier site participating in tissue-specific and systemic immunity. Oral Dis. (2018) 24:225. doi: 10.1111/odi.12729

3. Anderson MS, Venanzi ES, Chen Z, Berzins SP, Benoist C, Mathis D. The cellular mechanism of aire control of $t$ cell tolerance. Immunity. (2005) 23:227-39. doi: 10.1016/j.immuni.2005.07.005

4. Anderson MS, Venanzi ES, Klein L, Chen Z, Berzins SP, Turley SJ, et al. Projection of an immunological self shadow within the thymus by the aire protein. Science. (2002) 298:1395-401. doi: 10.1126/science.1075958r

5. Constantine GM, Lionakis MS. Lessons from primary immunodeficiencies: autoimmune regulator and autoimmune polyendocrinopathycandidiasis-ectodermal dystrophy. Immunol Rev. (2019) 287:10320. doi: 10.1111/imr.12714

6. Mathis D, Benoist C. Aire. Annu Rev Immunol. (2009) 27:287312. doi: 10.1146/annurev.immunol.25.022106.141532

7. Oftedal BE, Hellesen A, Erichsen MM, Bratland E, Vardi A, Perheentupa $\mathrm{J}$, et al. Dominant mutations in the autoimmune regulator aire are associated with common organ-specific autoimmune diseases. Immunity. (2015) 42:1185-96. doi: 10.1016/j.immuni.2015.04.021

8. Rosatelli MC, Meloni A, Meloni A, Devoto M, Cao A, Scott HS, et al. A common mutation in sardinian autoimmune polyendocrinopathycandidiasis-ectodermal dystrophy patients. Hum Genet. (1998) 103:42834. doi: 10.1007/s004390050846

9. Zlotogora J, Shapiro MS. Polyglandular autoimmune syndrome type i among iranian jews. J Med Genet. (1992) 29:824-6. doi: 10.1136/jmg.29.11.824

10. Ferre EM, Rose SR, Rosenzweig SD, Burbelo PD, Romito KR, Niemela JE, et al. Redefined clinical features and diagnostic criteria in autoimmune polyendocrinopathy-candidiasis-ectodermal dystrophy. JCI Insight. (2016) 1:88782. doi: $10.1172 /$ jci.insight. 88782

11. Ahonen P, Myllarniemi S, Sipila I, Perheentupa J. Clinical variation of autoimmune polyendocrinopathy-candidiasis-ectodermal dystrophy (apeced) in a series of 68 patients. N Engl J Med. (1990) 322:1829-36. doi: 10.1056/NEJM199006283222601

12. Suckling GW. Developmental defects of enamel-historical and presentday perspectives of their pathogenesis. Adv Dent Res. (1989) 3:8794. doi: 10.1177/08959374890030022901

13. Chang CC, Steinbacher DM. Treacher collins syndrome. Semin Plast Surg. (2012) 26:83-90. doi: 10.1055/s-0032-1320066

14. da Silva Dalben G, Costa B, Gomide MR. Prevalence of dental anomalies, ectopic eruption and associated oral malformations in subjects with treacher collins syndrome. Oral Surg Oral Med Oral Pathol Oral Radiol Endod. (2006) 101:588-92. doi: 10.1016/j.tripleo.2005.07.016

15. Ong KR, Visram S, McKaig S, Brueton LA. Sensorineural deafness, enamel abnormalities and nail abnormalities: a case report of heimler syndrome in identical twin girls. Eur J Med Genet. (2006) 49:18793. doi: 10.1016/j.ejmg.2005.07.003

16. Pollak C, Floy M, Say B. Sensorineural hearing loss and enamel hypoplasia with subtle nail findings: another family with heimler's syndrome. Clin Dysmorphol. (2003) 12:55-8. doi: 10.1097/00019605-200301000-00010

17. de la Pena VA, Valea MC. Treatment of enamel hypoplasia in a patient with usher syndrome. J Am Dent Assoc. (2011) 142:938-41. doi: 10.14219/jada.archive.2011.0300
18. Chascsa DM, Ferre EMN, Hadjiyannis Y, Alao H, Natarajan M, Quinones $\mathrm{M}$, et al. Apeced-associated hepatitis: clinical, biochemical, histological and treatment data from a large predominantly american cohort. Hepatology. (2020) 73:1088-104. doi: 10.1002/hep.31421

19. Pavlic A, Waltimo-Siren J. Clinical and microstructural aberrations of enamel of deciduous and permanent teeth in patients with autoimmune polyendocrinopathy-candidiasis-ectodermal dystrophy. Arch Oral Biol. (2009) 54:658-65. doi: 10.1016/j.archoralbio.2009.03.009

20. Purvis RJ, Barrie WJ, MacKay GS, Wilkinson EM, Cockburn F, Belton NR. Enamel hypoplasia of the teeth associated with neonatal tetany: a manifestation of maternal vitamin-d deficiency. Lancet. (1973) 2:8114. doi: 10.1016/S0140-6736(73)90857-X

21. Seow WK, Humphrys C, Tudehope DI. Increased prevalence of developmental dental defects in low birth-weight, prematurely born children: a controlled study. Pediatr Dent. (1987) 9:221-5.

22. Seow WK. Clinical diagnosis of enamel defects: pitfalls and practical guidelines. Int Dent J. (1997) 47:17382. doi: 10.1002/j.1875-595X.1997.tb00783.x

23. Seow WK. Enamel hypoplasia in the primary dentition: a review. ASDC J Dent Child. (1991) 58:441-52.

24. Crystal YO, Niederman R. Evidence-based dentistry update on silver diamine fluoride. Dent Clin North Am. (2019) 63:4568. doi: 10.1016/j.cden.2018.08.011

25. Yengopal V, Mickenautsch S. Caries preventive effect of casein phosphopeptide-amorphous calcium phosphate (cpp-acp): a meta-analysis. Acta Odontol Scand. (2009) 67:321-32. doi: 10.1080/00016350903160563

26. Rautemaa R, Richardson M, Pfaller M, Koukila-Kahkola P, Perheentupa J, Saxen H. Decreased susceptibility of candida albicans to azole antifungals: a complication of long-term treatment in autoimmune polyendocrinopathycandidiasis-ectodermal dystrophy (apeced) patients. J Antimicrob Chemother. (2007) 60:889-92. doi: 10.1093/jac/dkm299

27. Bruserud O, Costea DE, Laakso S, Garty BZ, Mathisen E, Makitie A, et al. Oral tongue malignancies in autoimmune polyendocrine syndrome type 1. Front Endocrinol (Lausanne). (2018) 9:463. doi: 10.3389/fendo.2018. 00463

28. Break TJ, Oikonomou V, Dutzan N, Desai JV, Swidergall M, Freiwald T, et al. Aberrant type 1 immunity drives susceptibility to mucosal fungal infections. Science. (2021) 371:eaay5731. doi: 10.1126/science.aay5731

29. Rautemaa R, Hietanen J, Niissalo S, Pirinen S, Perheentupa J. Oral and oesophageal squamous cell carcinoma-a complication or component of autoimmune polyendocrinopathy-candidiasis-ectodermal dystrophy (apeced, aps-i). Oral Oncol. (2007) 43:607-13. doi: 10.1016/j.oraloncology.2006. 07.005

30. Rosa DD, Pasqualotto AC, Denning DW. Chronic mucocutaneous candidiasis and oesophageal cancer. Med Mycol. (2008) 46:8591. doi: 10.1080/13693780701616023

Conflict of Interest: The authors declare that the research was conducted in the absence of any commercial or financial relationships that could be construed as a potential conflict of interest.

Copyright (C) 2021 Brenchley, Ferré, Schmitt, Gardner, Lionakis and Moutsopoulos. This is an open-access article distributed under the terms of the Creative Commons Attribution License (CC BY). The use, distribution or reproduction in other forums is permitted, provided the original author(s) and the copyright owner(s) are credited and that the original publication in this journal is cited, in accordance with accepted academic practice. No use, distribution or reproduction is permitted which does not comply with these terms. 